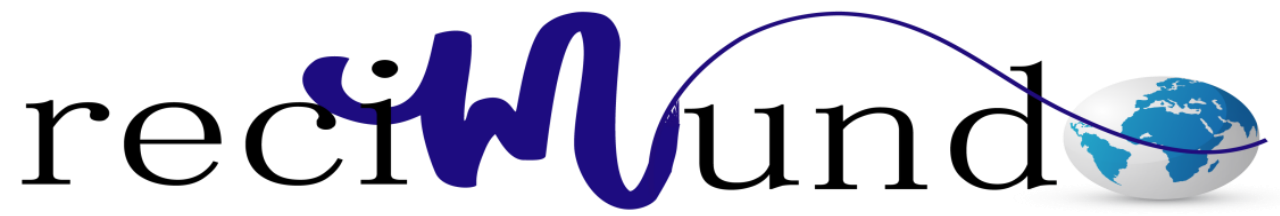

Revista Científica Mundo de la Investigación y el Conocimiento

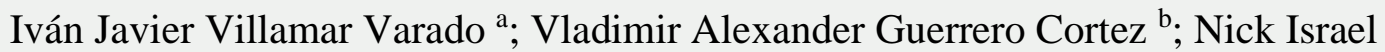
Balseca Villavicencio $^{\mathrm{c}}$

Plan estratégico para captar cuentas publicitarias para PYMES en la ciudad de Guayaquil

Revista Científica Mundo de la Investigación y el Conocimiento. Vol. 2 núm., especial, mayo, ISSN: 2588-073X, 2018, pp. 31-49

DOI: 10.26820/recimundo/2.esp.2018.31-49

Editorial Saberes del Conocimiento

Recibido: 13/12/2017

Aceptado: 14/02/2018

a. Universidad de Guayaquil; mba.ivanvillamar@gmail.com

b. Universidad de Guayaquil; vladimir.guerrerov@ug.edu.ec

c. Universidad de Guayaquil; nick.balsecav@ug.edu.ec 
Plan estratégico para captar cuentas publicitarias para pymes en la ciudad de Guayaquil

Vol. 2, núm. esp., (2018)

Iván Javier Villamar Varado;Vladimir Alexander Guerrero Cortez; Nick Israel Balseca Villavicencio

\section{RESUMEN}

La presente investigación denominada "Plan estratégico para captar cuentas publicitarias para PYMES, en la ciudad de Guayaquil" se realizó con el fin de crear estrategias para la captación de cuentas publicitarias para PYMES en la ciudad de Guayaquil. Esto se debe a la importancia que el manejo de la publicidad ha generado a través de los años para empresas de todos los sectores, los cuales han considerado que su presencia en las redes o por medio de la publicidad tradicional es vital para su posicionamiento en el mercado. Mediante la investigación de campo, focalizada a las PYMES de la ciudad de Guayaquil, se detectó la necesidad de aumentar la presencia de las marcas como una oportunidad para las entidades dedicadas a ofertar servicios especializados en publicidad, ya que, al tener un contrato con una empresa no solo se estima la realización de una venta de servicios sino una cuenta publicitaria que generará rentabilidad a largo plazo. Es por esto que, se proponen estrategias que permitan captar la atención de nuevos clientes y la inversión de un incremento en la capacidad productiva de la empresa a fin de satisfacer la demanda generada.

Palabras claves: Cuentas publicitarias, captación de clientes, planeación estratégica. 


\title{
Plan estratégico para captar cuentas publicitarias para pymes en la ciudad de \\ Guayaquil
}

Vol. 2, núm. esp., (2018)

Iván Javier Villamar Varado;Vladimir Alexander Guerrero Cortez; Nick Israel Balseca

Villavicencio

\begin{abstract}
The present research called "Strategic plan to capture advertising accounts for SMEs, in the city of Guayaquil" was carried out in order to create strategies for the capture of advertising accounts for SMEs in the city of Guayaquil. This is due to the importance that advertising management has generated over the years for companies from all sectors, which have considered that their presence in the networks or through traditional advertising is vital for their positioning in the market. market. Through field research, focused on SMEs in the city of Guayaquil, the need was detected to increase the presence of brands as an opportunity for entities dedicated to offer specialized services in advertising, since, having a contract with a The company not only estimates the realization of a sale of services but an advertising account that will generate longterm profitability. That is why, strategies are proposed to attract the attention of new customers and the investment of an increase in the productive capacity of the company in order to meet the demand generated.
\end{abstract}

Keywords: Advertising accounts, customer acquisition, strategic planning. 


\section{Plan estratégico para captar cuentas publicitarias para pymes en la ciudad de Guayaquil}

Vol. 2, núm. esp., (2018)

Iván Javier Villamar Varado; Vladimir Alexander Guerrero Cortez; Nick Israel Balseca Villavicencio

\section{Introducción.}

La publicidad es una herramienta de notable capacidad para llegar a los mercados y hacer conciencia entre la gente sobre diversos temas. Por lo tanto, muchos gobiernos e incluso organizaciones no gubernamentales (ONG) la utilizan con frecuencia para campañas de prevención de enfermedades, planificación familiar, uso inteligente de los recursos naturales, etc.

(Toca Torres, 2013)

Con seguridad las campañas de publicidad implican una gran inversión, sin embargo, si la estrategia es creativa y ejecutada de manera profesional, se logrará una experiencia digna de recordar en la mente de los consumidores con un impacto positivo para la compañía reflejado en el incremento de las ventas. (Pincheira Torres, 2013)

Así, es común encontrar espacios o ámbitos publicitarios donde se presenta la figura del público completamente repleto de comerciales. Algunos ejemplos de esos espacios o ámbitos pueden ser los medios de comunicación (a través de las propagandas) como también la misma acera o calle donde las publicidades están presentes por todos lados. Además, surgen espacios especialmente designados para el consumo de productos publicitados como son los shoppings, supermercados y negocios. (Baque Cantos, y otros, 2018)

También, la realización de planes estratégicos para la empresa destaca entre unos de los mayores recursos al inicio de un proyecto, con el fin de mantener la empresa dentro del mercado y direccionarla a su crecimiento. Es por esto que, se considera que todo emprendimiento debe 


\section{Plan estratégico para captar cuentas publicitarias para pymes en la ciudad de \\ Guayaquil}

Vol. 2, núm. esp., (2018)

Iván Javier Villamar Varado;Vladimir Alexander Guerrero Cortez; Nick Israel Balseca

Villavicencio

contar con estrategias o metas fijas, las cuales sirvan como una guía para la realización de sus funciones, no solo limitarse a la idea de vender el servicio y cubrir costos.

\section{Revisión de Literatura.}

Para una empresa institución pública o privada o PYMEs, sus clientes son el eje fundamental para determinar sus actividades, ya sea satisfaciendo a sus clientes actuales como captando a nuevos clientes que pueden ser un vínculo para que la empresa aumente su participación en el mercado en el cual se desarrolla. Muchos nuevos emprendimientos se generan de manera empírica, es decir, se realizan solo con la idea de prestar un servicio o dar un producto pero sin base en su ideología, maneras de captar clientes, procesos internos, entre otros.

La publicidad se basa en la idea o en la noción de que mientras más se realiza, permitirá incrementar el mercado objetivo, logrando que los consumidores se inclinen por el producto ofertado cuando tomen la decisión de compra.

Es importante para el cliente que su publicidad sea atractiva y provoque preferencias de los consumidores a su producto, lo cual plantea una diferencia sustancial. Lo que se busca es que el producto ofertado sea un producto reconocido, el cual ya ha sido probado y utilizado y que vuelve a ser elegido por aquel consumidor que lo obtiene. De este modo, la tarea de la publicidad es convertir a un producto, un objeto, un bien o un servicio en algo que se distingue del resto y que busca llegar a un determinado tipo de público.

Debido a esto se plantea la necesidad de generar un plan estratégico para la captación de cuentas publicitarias para una compañía de servicios integrales de diseño llamada PYMEs, la cual brinda 


\section{Plan estratégico para captar cuentas publicitarias para pymes en la ciudad de Guayaquil}

Vol. 2, núm. esp., (2018)

Iván Javier Villamar Varado; Vladimir Alexander Guerrero Cortez; Nick Israel Balseca Villavicencio

un conjunto de productos que puedan servir para los clientes, a fin de generar publicidad de alto impacto, cubriendo todas las áreas de la misma tales como: Fotografía, Eventos, Medios Impreso, Gigantografías, Banners, Roll-Up, Flyers, Brochures / Trípticos, Afiches, Revistas, Catálogos, Diseño de páginas web, Presencia y mantenimiento en redes sociales, Producción y Postproducción, Marca (Branding), Campaña Publicitaria, entre otros. Las PYMEs busca manejar los dos tipos de publicidad más significativa como son: Publicidad ATL, así como, Publicidad BTL. La presente investigación se justifica en la necesidad de demostrar la importancia que tiene la planificación estratégica en las empresas independientemente de su tamaño, considerando que, la formación de objetivos claros permite un crecimiento sostenible para todo tipo de emprendimientos, debido a que, se establecen acciones puntuales para la consecución de metas organizacionales. (Jiménez-Morales, 2016)

Debido a esto, el desarrollo de un emprendimiento, el cual busca aprovechar la necesidad de las PYMEs de recurrir a la publicidad en diversas formas para llegar a sus mercados objetivos para posicionarse en el mercado con servicios eficientes y procesos internos que permitan prestar la debida atención a cada cliente con resultados de calidad, a módicos precios y adaptables a cualquier industria que requiera sus servicios. La necesidad de la publicidad en el mercado actual. (Cristina, 2016)

Se la conoce como la divulgación de la información, al anunciar un nuevo producto o servicio o al actualizar la información, la imagen o la marca de una entidad transmitiendo información a través de este medio. Desde varios años atrás, la herramienta como la publicidad brinda grandes ventajas en los negocios grandes y pequeños, porque promueve una competencia justa y sana 


\section{Plan estratégico para captar cuentas publicitarias para pymes en la ciudad de \\ Guayaquil}

Vol. 2, núm. esp., (2018)

Iván Javier Villamar Varado;Vladimir Alexander Guerrero Cortez; Nick Israel Balseca

Villavicencio

entre los competidores personales y corporativos. Se ofrece algo interesante, desde cualquier cosa hasta una línea de producto o una campaña política, ya sea el medio utilizado por televisión radio o redes sociales.

La publicidad es otra forma de comunicación, representa la clave del éxito para la mayoría de las empresas. No hay que asumir que el consumidor está al tanto de los productos que la entidad o empresa tiene para ofertar, debido a que en la actualidad la competencia es muy tenas y las demás empresas probablemente están haciendo lo mismo y mostrando al mercado meta su producto que es diferente y mejor que cualquier otro.

Las empresas que a través de los medios tradicionales y digitales, son las que tienen un mayor grado de clientes, es decir cuentan con una cartera exclusiva de clientes y de esta forma hacen que el negocio prospere día a día. Los clientes, hoy en día, se inclinan por algo que les resulte familiar en lugar de algo que desconocen. Cuando uno trata de promocionar un producto nuevo, o extraño, siempre existe un mayor riesgo de aceptación, así como de precio y calidad. Por ende la publicidad ayuda a ganar confianza y poder jugar con la mente de los consumidores.

Estructura del plan de negocios

Para el éxito en un plan de negocios consiste en una serie de actividades que se relacionan entre sí para el desarrollo de una empresa, Brinda la facilidad de la creación o en su defecto, del crecimiento de una empresa (Fleitman, 2000). Hay que tomar en cuenta los siguientes puntos para el inicio de un proyecto: 


\section{Plan estratégico para captar cuentas publicitarias para pymes en la ciudad de Guayaquil}

Vol. 2, núm. esp., (2018)

Iván Javier Villamar Varado;Vladimir Alexander Guerrero Cortez; Nick Israel Balseca Villavicencio

- Misión: El propósito de una organización se define en la misión de la misma (Ferrel y Geoffrey Hirt, 2004).

- Visión: La visión es el camino a seguir por la organización a largo plazo, trata de orientar a la entidad estratégicamente al crecimiento de la misma y encaminarla a la competitividad" (Jack Fleitman, 2010).

- Objetivo General: Representa el fin concreto basado en la formulación del problema (Arias, 2006). - Objetivos específicos: Estos se derivan del objetivo general y ayudan a lograr el objetivo general, en él se indica conceptos, variables o dimensiones (Arias, 2006). • Descripción de productos o servicios: En este grupo se venden a los mismo clientes, comercializando en los diferentes tipos de venta en ciertos rangos de precios (Philip Kotler, 2012).

- Cadena de valor: Cada empresa representa un conjunto de actividades en producir, diseñar y entregar productos, las actividades están relacionadas con la cadena de valor.

Es primordial una evaluación estratégica del negocio a través de la matriz FODA que determina las fortalezas, oportunidades, debilidades y amenazas de una empresa (Díaz de Santos, 2009). Entre otros factores a tomar en cuenta, es la definición de los productos que se encuentran a su cargo, el mercado en el que está incursionando así como los clientes y competidores, a continuación se explica cada uno de ellos:

- Producto: Corresponde al conjunto de atributos como color, calidad, marca, se incluyen los servicios así como la reputación del vendedor, este puede ser un servicio, una persona, una idea o hasta un lugar (Stanton, Etzel y Walker, 2008). 


\section{Plan estratégico para captar cuentas publicitarias para pymes en la ciudad de}

Guayaquil

Vol. 2, núm. esp., (2018)

Iván Javier Villamar Varado;Vladimir Alexander Guerrero Cortez; Nick Israel Balseca

Villavicencio

- Mercado: Corresponde al conjunto de compradores potenciales así como de vendedores de una entidad organizacional, el cual se encuentra en un lugar geográfico, la empresa se encarga de localizarlo para poder ofrecer y vender los productos y servicios (Corrales, Carmen Castro, 2010).

- Clientes: Una persona, una empresa, o un hogar el cual proporciona de un ingreso correspondientes al costo que conlleva la captura de la atención, de vender y de brindar un servicio (Kotler Philip, K. L, 2006).

- Posición tecnológica: Corresponde a etapas técnicas, industriales y comerciales, todo este conjunto promueven el lanzamiento exitoso de productos manufacturados, en su defecto a la implementación de nuevos procesos técnicos (Hidalgo, P. e, 2009).

- Recursos operativos: Corresponden a los artículos y servicios que hacen falta para la administración del negocio. En estas áreas se incluye el bien capital como las computadoras, los suministros de oficina, reparación y de operación, así como los viajes y entretenimiento (Amor, Daniel, 2006).

- Competidores: Para que una empresa tenga éxito, esta debe de proporcionar toda la información necesaria en cuanto a sus productos o servicios hacia los competidores para su plena satisfacción. Debe ir más allá que simplemente adaptarse al medio.

- Factores claves de éxito: Se debe conocer a profundidad el negocio, conocer cuales productos o servicios dominan el negocio para crear una ventaja competitiva. Cuando se termina de elaborar el plan estratégico, se empieza con desarrollar el plan de marketing, según Porter, se considera el nivel de intensidad de un sector y la rentabilidad que este produce sobre dicho sector (Porter, 2012). 


\section{Plan estratégico para captar cuentas publicitarias para pymes en la ciudad de Guayaquil}

Vol. 2, núm. esp., (2018)

Iván Javier Villamar Varado; Vladimir Alexander Guerrero Cortez; Nick Israel Balseca Villavicencio

Se analiza un sector determinado como de producción, de distribución, de consumo de bienes, servicios o productos de un país. Cada año se diseña el plan de negocios a través de la publicidad que manejan la mayoría de las empresas que constan como carretas, locales, calles principales, entre otros.

El modelo de las cinco fuerzas, enseña cinco factores de una industria, los cuales son necesarios conocerlos y aprender a controlarlos para poder macar una pauta en el mercado y poder sobrevivir en el mismo para tomar una decisión acertada que lleven a la rentabilidad del mismo. (TERRY PORTER, 2012)

Los lazos de confianza y afectividad que se crean, impulsan los factores del éxito del negocio, escucha necesidades y opiniones para lograr cambios positivos y duraderos.

El atractivo sectorial permite establecer si un segmento es el que ofrece un sólido potencial que conlleva ganancias para el negocio (Sánchez, 2009). Cuando los consumidores tienen necesidades comunes a los que ofrece la empresa se los denomina mercado meta (Armstrong, $\mathrm{P}$. K, 2009).

Las estrategias de marketing conllevan la dirección y alcance de la empresa, se prevé de ventajas para la organización por medio de recursos que día a día están cambiando, y hace frente a las necesidades para cumplir expectativas.

Los objetivos de ventas consisten en vender lo que se hacen en lugar de hacer lo que el mercado desea, estos precios se fijan de acuerdo al vendedor y al consumidor (Philip Kotler, 2012). 


\section{Plan estratégico para captar cuentas publicitarias para pymes en la ciudad de \\ Guayaquil}

Vol. 2, núm. esp., (2018)

Iván Javier Villamar Varado;Vladimir Alexander Guerrero Cortez; Nick Israel Balseca

Villavicencio

El slogan es otro punto a tomar en cuenta, este y el logotipo son instrumentos distintivos para la estrategia comercial, además es importante un mecanismo de protección del consumidor (Noriega, 2010). También se debe tomar en cuenta la publicidad por la cual se transmitirá el mensaje por medio de ideas innovadoras. Estas ideas se pueden transmitir mediante debates ideológicos, mensajes publicitarios, entre otros.

El canal de distribución conllevan las empresas o individuos que logren facilitar que el producto sea factible, desde el fabricante hasta el consumidor final (keller y kotler, 2008). Para atender a los consumidores presenciales, se los realiza a través del punto de venta.

La estructura organizacional de una empresa es al análisis estratégico y el plan de marketing. La administración de Recursos Humanos se encarga de la planeación, organización, del control, que permite alcanzar los objetivos individuales, estos están relacionados de manera directa e indirecta.

Un buen plan para el manejo del recurso humano es la puesta en práctica de manera sistemática, la provisión y demanda de cada uno de los empleados de una empresa.

Cuando se establece el número de empleados y el tipo, es necesario que el planear las labores de reclutamiento, la selección, capacitación y otros puntos más (Werther, William B. Jr., 2000).

Planificación estratégica

Para conceptualizar el término "Planeación Estratégica", se debe enfocar en el logro de los objetivos de la empresa por medio de la formulación, implementación y evaluación de las 


\section{Plan estratégico para captar cuentas publicitarias para pymes en la ciudad de Guayaquil}

Vol. 2, núm. esp., (2018)

Iván Javier Villamar Varado;Vladimir Alexander Guerrero Cortez; Nick Israel Balseca Villavicencio

decisiones de la organización. Este término se originó en los 1950s, pero tomo fuerza en el ámbito empresarial entre los años 1960s y 1970s. Las compañías tomaron a la planificación estratégica como la solución a los múltiples problemas que las organizaciones presentaban y comenzaron a depender de ella de manera obsesiva. En los años 1980s, algunos modelos de planeación no resultaron efectivos y poco a poco fueron descartados. Con el paso del tiempo, en los años 1990s se concreta el resurgimiento de la planificación estratégica llegando a lo que es, en la actualidad. (David, 2011)

La planeación estratégica comprende los planes de los altos mandos de una organización para obtener resultados acordes a la misión y objetivos de la compañía.

También se puede analizar como una serie de pasos que los altos mandos de una empresa deben lograr para poder establecer metas y objetivos claros en una organización, por ejemplo: analizar las oportunidades y amenazas que ofrece el ambiente externo de la compañía, analizar las debilidades y fortalezas que la compañía demuestra en su ambiente interno, establecer la misión y visión de la empresa y el desarrollo de sus objetivos, formular estrategias fusionando las oportunidades y amenazas con las debilidades y fortalezas del ambiente, implementar dichas estrategias y llevar un control de estas para garantizar que las metas de la empresa son atendidas. (Wringht, Kroll, \& Parnell, 1996)

\section{El neuromarketing}

El neuromarketing consiste en la combinación de dos disciplinas: las neurociencias y el marketing. De este modo, permite usar herramientas y técnicas de las primeras a la toma de 


\section{Plan estratégico para captar cuentas publicitarias para pymes en la ciudad de Guayaquil}

Vol. 2, núm. esp., (2018) Iván Javier Villamar Varado;Vladimir Alexander Guerrero Cortez; Nick Israel Balseca

Villavicencio

decisiones en el ámbito de las estrategias de marketing, analizando así como afectan al consumidor los estímulos publicitarios (conscientes o inconscientes) a nivel de memoria, atención y emoción. Dicho de otro modo, el neuromarketing estudia el funcionamiento del cerebro aplicado al ámbito de las decisiones de compra (o de uso).

Al fin y al cabo, dichas decisiones se basan en sensaciones subjetivas fundamentadas en estímulos sensoriales. Estímulos de los que muchas veces los consumidores ni siquiera son conscientes y, por ello, ni los más exhaustivos estudios de mercado pueden recoger.

Éstos tampoco tienen en cuenta los casos en que el sujeto responde a una encuesta motivado por la situación o por su interlocutor, aunque realmente no lo piense. Es decir, las técnicas que las neurociencias aportan al neuromarketing (mediciones psicofisiológicas periféricas y centrales relativas a la actividad cerebral, el ritmo cardíaco, respuesta galvánica de la piel, movimiento ocular, etc) permiten evaluar al consumidor por cómo reacciona su cerebro, y no por lo que voluntariamente nos comunica.

Es fácil encontrar información en Internet con aproximaciones al neuromarketing basadas en mitos y falacias, tanto a favor como en contra del mismo. Por otra parte, gente que lo considera poco menos que una herramienta de manipulación de masas propio de películas de cienciaficción. Dos planteamientos en el fondo bastante similares que chocan con una tercera postura: la que considera que el sufijo neuro- se convierte en mero encubrimiento de estafas en cuanto se le saca del ámbito más vinculado a la investigación biomédica. 


\section{Plan estratégico para captar cuentas publicitarias para pymes en la ciudad de Guayaquil}

Vol. 2, núm. esp., (2018)

Iván Javier Villamar Varado;Vladimir Alexander Guerrero Cortez; Nick Israel Balseca Villavicencio

Se plantea que existen seis grandes objetivos del neuromarketing, según lo menciona Alba Ferrer de la Universidad Abat Oliba CEU en el paper "Neuromarketing: la tangibilización de las emociones":

- Mediante los estímulos que el sistema nervioso envía, percibir si el individuo recibe cada día en lenguaje cerebral y el procesamiento de los mismos.

- En necesario realizar un estudio de los mensajes publicitarios.

- Realizar una selección adecuada del formato de conexión emocional hacia el consumidor.

- Se tiene que estudiar la mente del consumidor para poder descifrar su posible conducta futura.

- Seguir adecuadamente todos los aspectos del marketing, es decir, producto, precio, promociones, etc.

- Se debe mejorar la planificación estratégica del branding en son de poder prever las verdaderas necesidades y expectativas del consumidor.

\section{Metodología.}

En el presente trabajo investigativo se utilizaron tres tipos de métodos de investigación:

Método Analítico.-

El método analítico toma por punto de partida el conocimiento buscado, y retrocede, suponiendo ordenes de dependencia en los que ese conocimiento está incluido, hasta lograr la conexión con cadenas deductivas que son ya conocidas y evidentes. (Caimi, 2003, pág. 39). Es el método que será utilizado en el momento del procesamiento de la información que ha sido recopilada durante 


\section{Plan estratégico para captar cuentas publicitarias para pymes en la ciudad de \\ Guayaquil}

Vol. 2, núm. esp., (2018)

Iván Javier Villamar Varado;Vladimir Alexander Guerrero Cortez; Nick Israel Balseca

Villavicencio

la investigación del trabajo, la misma que nos permitirá extraer los elementos fundamentales para fundamentación del trabajo.

Método deductivo.-

"Es un método de razonamiento que consiste en tomar conclusiones generales para explicaciones particulares". (Bernal, 2006, pág. 56). Este método será utilizado en la elaboración de las conclusiones que nos permitirá determinar los resultados del proceso de investigación y en las recomendaciones para poder proyectarnos al futuro.

Método Estadístico.-

"El método estadístico, dentro del método científico, consiste en una serie de pasos para llegar al verdadero conocimiento estadístico". (García, Ramos, \& Ruiz, 2006, pág. 6). Este método lo utilizamos al momento de recopilar la información para la elaboración de los resultados con la que podemos realizar la tabulación y elaboración de los cuadros estadísticos.

Tipo de investigación

En el trabajo de investigación se seleccionaron diferentes tipos de investigación:

Investigación documental.-

Mediante la investigación documental se recopila la información de las correspondientes variables, con el objetivo de efectuar el debido análisis para diseñarlo en el marco teórico, que son las claves fundamentales para procesar la información. 


\section{Plan estratégico para captar cuentas publicitarias para pymes en la ciudad de Guayaquil}

Vol. 2, núm. esp., (2018)

Iván Javier Villamar Varado; Vladimir Alexander Guerrero Cortez; Nick Israel Balseca Villavicencio

Investigación de campo.-

Esta investigación de campo ayuda a averiguar los posibles problemas que existen al implementar el sistema y a evaluar la situación para buscar las mejores alternativas en dar solución a tiempo a los inconvenientes que se puedan presentar.

Investigación explicativa.-

Por medio de esta investigación se logra interpretar y entender la naturaleza de los resultados que surgirán de las encuestas, para dar un análisis que fundamentará el marco investigativo.

\section{Técnicas de investigación}

Para la recolección de datos necesarios para el presente proyecto se realizarán los presentes datos:

Tabulación de información: Permite obtener resultados que indiquen las carencias y fortalezas de la institución.

Interpretación gráfica de resultados: Una vez obtenidos los resultados de cada una de las interrogantes planteadas en la encuesta se hizo uso de gráficos y tablas para que la información presente sea fácilmente comprendida.

Análisis de la información: Finalmente, se analizaron cada uno los resultados obtenidos para generar una conclusión individual; estas conclusiones permiten establecer una conclusión general. (Rodriguez, 2005). 


\section{Plan estratégico para captar cuentas publicitarias para pymes en la ciudad de \\ Guayaquil}

Vol. 2, núm. esp., (2018)

Iván Javier Villamar Varado;Vladimir Alexander Guerrero Cortez; Nick Israel Balseca

Villavicencio

\section{Población y muestra}

\section{Población o Universo}

Con la finalidad de poder conocer el mercado en el cual se desarrollará el negocio se realizará un estudio de campo, para la recolección de datos dentro de la población objetivo. Para ello, se utilizará la herramienta conocida como encuesta, aplicada a una muestra del total de personas que componen los clientes potenciales, utilizando la siguiente fórmula:

\section{Formulas}

\section{Muestra}

Dicho cálculo del tamaño de la muestra, se estimó un nivel de confianza del 96\%, en donde Z toma valor de 1.96 debido al porcentaje de confianza, también se consideró un $4 \%$ para un margen de error, un 50\% para la probalidad de éxito y el otro 50\% para la probabilidad de fracaso, para la cual se tomara como población objetivo a la totalidad de PYMEs existentes en Guayaquil.

Según datos del INEC, existen un total de 842.936 PYMEs en el país para el año 2016, de las cuales el 19,37\% se ubican en la provincia del Guayas, dando un total de 163.276 PYMEs, de las cuales, 97.374 se encuentran en la ciudad de Guayaquil, lo cual es la población objeto de estudio, dando una muestra de 383 empresas encuestadas

\section{Formulas}




\section{Plan estratégico para captar cuentas publicitarias para pymes en la ciudad de Guayaquil}

Vol. 2, núm. esp., (2018)

Iván Javier Villamar Varado;Vladimir Alexander Guerrero Cortez; Nick Israel Balseca Villavicencio

\section{Resultados}

Con los resultados obtenidos de las encuestas realizadas se detectó la necesidad de aumentar la presencia de las marcas como una oportunidad para las entidades dedicadas a ofertar servicios especializados en publicidad, ya que, al tener un contrato con una empresa no solo se estima la realización de una venta de servicios sino una cuenta publicitaria que generará rentabilidad a largo plazo.

\section{Conclusión.}

Como conclusiones finales del presente proyecto se establecen los siguientes puntos:

Las estrategias para la captación de clientes se basan en la creación de actividades que permitan diferenciar a la empresa de sus competidores, considerando la dinámica del sector en el que se desarrolla. No solo se establece la necesidad de ofertar mejores precios, puesto que, siempre este punto se limita a los costos fijos y variables de la empresa y la dinámica de la industria. Sino que se debe dar un valor agregado focalizado en un buen servicio, agilidad en la atención, ofertas y promociones, entre otros aspectos que logren que el cliente potencial seleccione a la entidad.

El cliente potencial de las PYMEs se establece como las pequeñas y medianas empresas, a pesar de que no se descartar captar mercado en las grandes empresas.

Debido al fomento de la cultura organizacional que tiene lugar en el país, las PYMES consideran cada vez más indispensable el desarrollo de estrategias de marketing, lo cual es un mercado 


\section{Plan estratégico para captar cuentas publicitarias para pymes en la ciudad de \\ Guayaquil}

Vol. 2, núm. esp., (2018)

Iván Javier Villamar Varado;Vladimir Alexander Guerrero Cortez; Nick Israel Balseca

Villavicencio

atractivo y potencial para la entidad que puede ser aprovechado antes que el mercado sea saturado al ser evidente el potencial del negocio.

Con el fin de ampliar la cartera de clientes de las PYMEs, se estima la necesidad de plantear diversas estrategias como la restructuración del logo, la creación de base de datos para manejar la publicidad en clientes potenciales, estrategias de crecimiento basadas en la diferenciación con la competencia y la ampliación de la capacidad productiva para ajustarse a la nueva demanda.

\section{Bibliografía.}

Baque Cantos, M., Cañarte Quimis, L., Santistevan Villacreses, K., Sumba Bustamante, R., Loor Carvajal, V., \& Cantos Figueroa, M. (2018). Marketing para los empresarios y emprendedores del Ecuador. Quito: MAWIL.

Cristina, A. (2016). Relaciones públicas 2.0. Cómo gestionar la comunicación corporativa en el entorno digital. Mexico: ACED.

DELLEMC. (Agosto de 2017). www.emc.com. Obtenido de https://www.emc.com/collateral/technical-documentation/h15104-vxrail-appliancetechbook.pdf

Fleitman, J. (2000). Negocios exitosos : cómo empezar, administrar y operar eficientemente un negocio. Mexico: McGraw-Hill Interamericana.

IDC Custom Solutions. (Diciembre de 2016). Digital Transformation Drives New IT Infraestructure Requirements. Framingham.

Jiménez-Morales, M. (2016). Relaciones públicas y redes sociales: creación e implementación de marcas personales. Opción, 32(9), 945-960.

Pincheira Torres, I. (2013). Entre el marketing empresarial y la política pública estatal: el gobierno de la felicidad en el neoliberalismo chileno. Revista Latinoamericana de Estudios sobre Cuerpos, Emociones y Sociedad, 5(11), 7-20.

TERRY PORTER, R. (2012). Sustainability and Business in a Complex World. ROBBIN DERRY, 117(1), 33-53. 
Plan estratégico para captar cuentas publicitarias para pymes en la ciudad de Guayaquil

Vol. 2, núm. esp., (2018)

Iván Javier Villamar Varado;Vladimir Alexander Guerrero Cortez; Nick Israel Balseca Villavicencio

Toca Torres, C. (2013). El marketing al servicio de las organizaciones del tercer sector. Estudios Gerenciales, 29(129), 386-395. 